\title{
Burnout among paediatric residents during the COVID-19 outbreak in France
}

\author{
Ludovic Treluyer $^{1,2}$ (D) Pierre Tourneux ${ }^{1,2}$
}

Received: 13 August 2020 / Revised: 19 November 2020 / Accepted: 10 December 2020 / Published online: 7 January 2021

(C) The Author(s), under exclusive licence to Springer-Verlag GmbH, DE part of Springer Nature 2021

\begin{abstract}
The primary objective of the study was to assess the prevalence of burnout among paediatric residents during the coronavirus disease 2019 (COVID-19) outbreak in France. The secondary objective was to identify risk factors associated with burnout in this population. In a nationwide, cross-sectional survey, a questionnaire was e-mailed to all paediatric residents in France in the first week of May 2020. The prevalence of burnout was assessed with the validated French-language version of the Maslach Burnout Inventory - Human Services Survey. The questionnaire also contained items on the residents' sociodemographic characteristics and professional situation. Three hundred and forty paediatric residents completed the questionnaire. The median age was 27 (interquartile range 25-28) and $285(83.8 \%$, 95\% confidence interval (CI) [79.5-87.6]) of the residents were women. The prevalence of burnout was $37.4 \%, 95 \% \mathrm{CI}$ [32.2-42.7]. There was no association between burnout and exposure to the consequences of COVID-19, which may be related to the low incidence of severe COVID-19 among children. In contrast, the hours worked per week and the anxiety scores were significantly associated with burnout.

Conclusion: The level of burnout among French paediatric residents is a matter of concern for residents, and cannot be ascribed to the COVID-19 outbreak. Preventive actions should be implemented, with a reduction in working hours and support programs to help manage work-related anxiety.
\end{abstract}

What is Known:

- Burnout is a concern for both residents and the patients they care for.

- Natural disasters disrupt the health care organizations and increase the burnout rate.

What is New:

- The prevalence of burnout among paediatric residents in France is 37.4\%, 95\% CI [32.2-42.7].

- COVID-19 outbreak is not associated with burnout in this population but anxiety and working hours per week might be modifiable risk factors.

Keywords Anxiety $\cdot$ Burnout $\cdot$ COVID-19 $\cdot$ Paediatric resident $\cdot$ Working hours

\section{Abbreviations}

CI Confidence interval

COVID-19 Coronavirus disease 2019

Communicated by Daniele De Luca

Ludovic Treluyer

1.treluyer@gmail.com

Pierre Tourneux

tourneux.pierre@chu-amiens.fr

1 Paediatric Intensive Care Unit, CHU Amiens-Picardie, Amiens, France

2 PériTox UMR I 01, UFR de Médecine, Jules Verne University of Picardie, Amiens, France

$\begin{array}{ll}\text { MBI - HSS } & \begin{array}{l}\text { Maslach Burnout Inventory - Human } \\ \text { Services Survey }\end{array} \\ \text { IQR } & \text { Interquartile range }\end{array}$

\section{Introduction}

Physicians are at high risk of psychological disorders including burnout $[1,2]$. According to the World Health Organization's International Statistical Classification of Diseases and Related Health Problems, 11th Revision [3], burnout is "a syndrome conceptualized as resulting from chronic workplace stress that has not been successfully managed". The condition is characterized by emotional exhaustion, depersonalization, and reduced personal accomplishment 
[4]. Burnout is known to have very harmful effects on physician health, patient care, and health care systems; the effects respectively include (i) substance abuse, depression, and suicidal ideation, (ii) medical errors, longer recovery times, and lower patient satisfaction, and (iii) reduced physician productivity and increased physician turnover for health care system [5].

The 2020 pandemic of coronavirus disease 2019 (COVID19) disrupted health care systems and work organizations across the world and notably in France. The outbreak resulted in greater workloads and increased levels of stress and fatigue among physicians [6]. Before the COVID-19 outbreak, the prevalence of burnout among paediatric residents was already high (31 to $40 \%$ in the USA; no data for European countries) [7-9]. By disrupting the health care system, the COVID-19 outbreak may have increased this prevalence [6]. Increases in (i) stress levels related to contact with COVID-19 patients, (ii) the workload associated with more frequent medical consultations in paediatric emergency departments, and (iii) physicians' sick leave might also be responsible for greater burnout among paediatric residents.

We reasoned that better epidemiological knowledge of (i) burnout among paediatric residents and (ii) the impact of the current COVID-19 outbreak would make it possible to implement preventive actions and adapt them if further outbreaks occur [10].

Hence, the primary objective of the present study was to assess the national prevalence of burnout among paediatric residents in France during the COVID-19 breakout. The secondary objective was to identify factors associated with burnout in this population.

\section{Method}

\section{Study design and population}

We performed a nationwide, cross-sectional survey of paediatric residents affiliated with all 28 French university hospitals teaching paediatrics. We calculated that around 1300 residents were eligible for the study; this number was based on the number of new paediatric residents in France in $2019(n=$ 325 ) and the duration of the residency in paediatrics (4 years). An invitation to participate in the study (with a link to an online questionnaire) was e-mailed to each resident in the first week of May 2020 (i.e. during the national lockdown period). A reminder was sent 3 days after the first e-mail. Replies were accepted up until May 11, 2020 (the last day of the national lockdown period). We chose to stop collecting data at the end of the lockdown so that major societal change did not bias our interpretation of the results. The e-mail informed the residents about the study's objectives and invited them to click on the link if they wished to participate in the study. The online questionnaire's homepage provided information about the group conducting the study and how the participants' data would be processed. Residents participating in the study were informed of their data protection rights, in line with French legislation and the European Union's General Data Protection Regulation.

The online questionnaire contained items on the residents' sociodemographic characteristics, professional situation, and burnout. Respondents were also asked to state their level of anxiety before the epidemic and then during the epidemic on a numerical scale [11] ranging from 0 (no anxiety) to 100 (highest level imaginable).

The study protocol was approved by an independent ethics committee (Amiens University Hospital, Amiens, France; reference: PI2020_843_0037 (2020-04-10)).

\section{Burnout definition}

The main outcome was the prevalence of burnout, as assessed with the French version of the Maslach Burnout Inventory Human Services Survey (MBI - HSS) [12]. This scale evaluates three domains of burnout in caregiver populations and the French version has been validated [13]. The questionnaire consists of 22 items, each of which is scored from 0 (never) to 7 (several times a day). Nine of the items assess emotional exhaustion, five assess depersonalization, and eight assess reduced personal accomplishment. The cut-off scores for the emotional exhaustion and depersonalization domains were respectively $\geq 30$ and $\geq 12$. The cut-off for reduced personal accomplishment was $\leq 33$. Residents were considered to have experienced burnout when the level of emotional exhaustion or depersonalization exceeded the cut-off, regardless of the presence or absence of reduced personal accomplishment $[14,15]$.

\section{Definition of the professional consequences of COVID- 19}

We assessed the association between the COVID-19 outbreak and burnout with regard to three different items. In order to study the impact of the epidemic on the resident's workload, the various regions of France were categorized according to the incidence of admissions to intensive care units for COVID-19 in the week before the questionnaire was sent out. The two other items were declarative. The residents had to answer the two following questions: "Did your professional activity change as a result of the COVID-19 outbreak?" and "Did you participate directly in the management of patients with COVID-19?"

\section{Statistical analyses}

Descriptive statistics were calculated after assessment of the data distribution using the Shapiro-Wilk test. Given that some variables were not normally distributed, the data were 
expressed as the median [interquartile range] and percentages were presented with their exact $95 \%$ binomial confidence intervals (CIs). The associations between burnout and the residents' sociodemographic and professional characteristics were assessed in bivariate analyses. Categorical variables were compared using a chi-square test, and median values were compared using a Wilcoxon test. Odds ratio were calculated with logistic regression coefficient. The threshold for statistical significance was set to $p<0.05$. All analysis was performed using R software (version 3.5.0) [16].

\section{Results}

Three hundred and forty paediatric residents $(26.1 \%$ of the 1300 in France) completed the questionnaire. The median (interquartile range [IQR]) age was 27 (IQR 25-28), 285 $(83.8 \%, 95 \% \mathrm{CI}[79.5-87.6])$ of the residents were women, and $242(71.2 \%, 95 \% \mathrm{CI}$ [66.0-75.9]) of the residents lived with a partner. The median number of night shifts per month and per resident was 4 (IQR 3-5) and $94(27.6 \%, 95 \%$ CI [23.0-23.7]) of the residents worked more than $60 \mathrm{~h}$ a week. With regard to COVID-19, 271 (79.7\%, 95\% CI [75.0-83.9]) of residents reported a change in their professional activity as a result of the outbreak, and $136(40.0 \%, 95 \%$ CI [34.8-45.4]) were directly involved in the management of patients with COVID-19 (Table 1). The median anxiety score was 40 (IQR 20-60) before the outbreak and 50 (IQR 30-60) during the outbreak. The prevalence of burnout in the study population was $37.4 \%, 95 \%$ CI [32.2-42.7]. The proportion of residents reporting a high level of emotional exhaustion was $23.5 \%, 95 \%$ CI [19.1-28.4], and 28.2\%, 95\%CI [23.5-33.3] reported a high level of depersonalization (Table 2).

In bivariate analyses, we found that sex, the number of hours worked per week, and the anxiety scores were associated with burnout (Table 3). There was no association between burnout and a change in activity due to COVID-19 outbreak, direct involvement in the management of patients with COVID-19, and the intensive care unit admission rate for COVID-19 in the resident's geographic area (Table 3). The emotional exhaustion (Appendix A) and depersonalization (Appendix B) domain scores were also associated with the working hours per week and the anxiety scores.

\section{Discussion}

The prevalence $[95 \% \mathrm{CI}]$ of burnout among paediatric residents in France was $37.4 \%$ [32.2-42.7]. This value is high and similar to those reported by studies in the USA (ranging from 31 to $40 \%[17,18])$, even though the countries' respective health systems are organized differently. The level of burnout among paediatric residents is nevertheless lower than among other specialties. In a recent meta-analysis [19], the proportion was $42.5 \%$ for residents in general surgery, anaesthesiology, orthopaedics, and obstetrics-gynaecology. One possible explanation is that paediatric residents are less likely to be confronted with emergency situations than the other residents mentioned - even though the population of paediatrics residents is heterogeneous, and some residents are exposed to emergency-related stress in neonatal or paediatric intensive care units. With regard to the prevalence rates for the MBI - HSS subdimensions, the value for depersonalization (corresponding to a negative, cynical attitude toward patients) was higher in our study than in previous studies [19]. A meta-analysis has reported that the prevalence rates depend on the specialty: the prevalence of depersonalization was higher among obstetrics-gynaecology residents, whereas the prevalence of emotional exhaustion was higher among cardiology residents [19].

The self-reported working hours might explain (at least in part) the prevalence of burnout among paediatric residents in France [18]. As described in the literature, we observed that the anxiety scores (both before and during the COVID-19 outbreak) were significantly associated with burnout $[17,18$, 20]. The prevalence of burnout was higher among male residents than among female residents, as also described in the literature [19]. In contrast to previous studies, we did not observe a significant association between burnout and the resident's level of training $(p<0.16)$ [21].

In the present study, the consequences of COVID-19 (the intensive care unit admission rate for COVID-19 in the resident's region, direct participation in the management of patients with COVID-19, and changes in professional activity due to the COVID-19 outbreak) were not significantly associated with burnout. Earlier studies have shown various associations between physician burnout and natural disasters [22-24]. After an earthquake in Italy in 2016, the prevalence of physician burnout was high (25.97\%) [22]. Similarly, the prevalence of physician burnout after Hurricane Irma in 2017 was $34.1 \%$ (95\% CI [28.2-40.0]) [24]. Yeo et al. focused on the responses of first-year trainee physicians to Hurricane Harvey in 2017 [23]. The researchers observed that the prevalence of emotional exhaustion was about $10.2 \%$ in their cohort. The lack of an impact of the COVID-19 outbreak in the present study might be due to the low incidence of severe disease in children [25] or the fact that the baseline prevalence of burnout in our study population was already high [17]. Similar results were observed by Dimitriu et al.; the burnout rate in resident in first-line contact with patients with suspected or confirmed COVID-19 was not higher than in other residents [26]. In Dimitriu et al.'s single-centre study, the prevalence of burnout among the residents was extremely high $(76 \%)$, which meant that there was little room for a further increase.

Our study had several limitations. Firstly, burnout can be defined in several ways. We only considered the three main 
Table 1 Characteristics of the study population

\begin{tabular}{|c|c|c|}
\hline & \multicolumn{2}{|c|}{ Population } \\
\hline & $n / N$ & $\%(95 \% \mathrm{CI})$ \\
\hline \multicolumn{3}{|l|}{ Sex, } \\
\hline Men & $55 / 340$ & $16.2(12.4-20.5)$ \\
\hline Women & $285 / 340$ & $83.8(79.5-87.6)$ \\
\hline Age, median (IQR) & 27 & $(25-28)$ \\
\hline \multicolumn{3}{|l|}{ Residency start year, } \\
\hline$\leq 2016$ & $106 / 340$ & $31.2(26.3-36.4)$ \\
\hline 2017 & $81 / 340$ & $23.8(19.4-28.7)$ \\
\hline 2018 & $74 / 340$ & $21.8(17.5-26.5)$ \\
\hline 2019 & $79 / 340$ & $23.2(18.8-28.0)$ \\
\hline \multicolumn{3}{|l|}{ Living status, } \\
\hline Living alone & $98 / 340$ & $28.8(24.1-34.0)$ \\
\hline Living with a partner & $242 / 340$ & $71.2(66.0-75.9)$ \\
\hline Number of night shifts per month, median (IQR) & 4 & $4(3-5)$ \\
\hline \multicolumn{3}{|l|}{ Hours worked per week, } \\
\hline$<48 \mathrm{~h}$ & $27 / 340$ & $8.0(5.3-11.3)$ \\
\hline $48-60 \mathrm{~h}$ & $219 / 340$ & $64.4(59.1-69.5)$ \\
\hline$>60 \mathrm{~h}$ & $94 / 340$ & $27.6(23.0-23.7)$ \\
\hline \multicolumn{3}{|l|}{ Change in professional activity due to the COVID-19 outbreak, } \\
\hline Yes & $271 / 340$ & $79.7(75.0-83.9)$ \\
\hline No & $69 / 340$ & $20.3(16.1-25.0)$ \\
\hline \multicolumn{3}{|l|}{ Direct involvement in the care of patients with COVID-19, } \\
\hline Yes & $136 / 340$ & $40.0(34.8-45.4)$ \\
\hline No & $204 / 340$ & $60.0(54.6-65.2)$ \\
\hline \multicolumn{3}{|l|}{ ICU admissions for COVID-19 per 100,000 inhabitants ${ }^{*}$} \\
\hline$[0-0.5]$ & $64 / 340$ & $18.8(14.8-23.4)$ \\
\hline$[0.5-1]$ & $108 / 340$ & $31.8(26.8-37.0)$ \\
\hline$[1-2]$ & $76 / 340$ & $22.4(18.0-27.2)$ \\
\hline$>2$ & $92 / 340$ & $27.0(22.4-32.1)$ \\
\hline Anxiety score before the COVID-19 outbreak, median (IQR) & 40 & $(20-60)$ \\
\hline Anxiety score during the COVID-19 outbreak, median (IQR) & 50 & $(30-60)$ \\
\hline
\end{tabular}

$I Q R$, interquartile range; $C I$, confidence interval

* Intensive care unit admission rate for COVID-19 per 100,000 inhabitants in the resident's geographic area of practice (April 22 to 28,2020 )

\begin{tabular}{|c|c|c|c|c|}
\hline \multirow[t]{2}{*}{ Burnout domains } & \multicolumn{2}{|c|}{ No burnout } & \multicolumn{2}{|l|}{ Burnout } \\
\hline & $\begin{array}{l}n / N \\
213 / 340\end{array}$ & $\begin{array}{l}\%(95 \% \mathrm{CI}) \\
62.6(57.2-67.8)\end{array}$ & $\begin{array}{l}n / N \\
127 / 340\end{array}$ & $\begin{array}{l}\%(95 \% \mathrm{CI}) \\
37.4(32.2-42.7)\end{array}$ \\
\hline High degree of emotional exhaustion & $260 / 340$ & $76.5(71.6-80.9)$ & $80 / 340$ & $23.5(19.1-28.4)$ \\
\hline High degree of depersonalization & $244 / 340$ & $71.8(66.7-76.5)$ & $96 / 340$ & $28.2(23.5-33.3)$ \\
\hline $\begin{array}{l}\text { High degree of reduced personal } \\
\text { accomplishment }\end{array}$ & $254 / 340$ & $74.7(69.7-79.2)$ & $86 / 340$ & $25.3(20.8-30.3)$ \\
\hline
\end{tabular}

$C I$, confidence interval

Residents were considered to have experienced burnout when either the level of emotional exhaustion or depersonalization exceeded the cut-off, regardless of the presence or absence of reduced personal accomplishment 
Table 3 Factors associated with burnout among paediatric residents in France

\begin{tabular}{|c|c|c|c|c|c|c|}
\hline & \multicolumn{2}{|c|}{ No burnout } & \multicolumn{2}{|c|}{ Burnout } & \multirow[t]{2}{*}{$\mathrm{OR}, 95 \% \mathrm{CI}$} & \multirow[t]{2}{*}{$p$ value } \\
\hline & $n / N$ & $\%(95 \% \mathrm{CI})$ & $n / N$ & $\%(95 \% \mathrm{CI})$ & & \\
\hline Sex & & & & & & 0.01 \\
\hline Men & $26 / 213$ & $12.2(8.1-17.4)$ & $29 / 127$ & $22.8(15.9-31.1)$ & 1 & \\
\hline Women & $187 / 213$ & $87.8(82.6-91.9)$ & $98 / 127$ & $77.2(68.9-84.1)$ & $0.47(0.26-0.84)$ & \\
\hline Age, median (IQR) & 27 & $(25-28)$ & 27 & $(25-28)$ & $1.00(0.88-1.14)$ & 0.97 \\
\hline Residency start year & & & & & & 0.44 \\
\hline$\leq 2016$ & $70 / 213$ & $32.9(26.6-39.6)$ & $36 / 127$ & $28.3(20.7-37.0)$ & 1 & \\
\hline 2017 & $46 / 213$ & $21.6(16.2-27.7)$ & $35 / 127$ & $27.6(20.0-36.2)$ & $1.48(0.82-2.69)$ & \\
\hline 2018 & $44 / 213$ & $20.6(15.4-26.7)$ & $30 / 127$ & $23.6(16.5-32.0)$ & $1.33(0.72-2.45)$ & \\
\hline 2019 & $53 / 213$ & $24.9(19.2-31.2)$ & $26 / 127$ & $20.5(13.8-28.5)$ & $0.95(0.51-1.77)$ & \\
\hline Living status & & & & & & 0.07 \\
\hline Living alone & $54 / 213$ & $25.4(19.7-31.7)$ & $44 / 127$ & $34.6(26.4-43.6)$ & 1 & \\
\hline Living with a partner & $159 / 213$ & $74.6(68.3-80.3)$ & $83 / 127$ & $65.4(56.4-73.6)$ & $0.64(0.40-1.04)$ & \\
\hline Number of night shifts per month, median (IQR) & 4 & $(3-5)$ & 4 & $(3-5)$ & $1.08(0.95-1.24)$ & 0.24 \\
\hline Hours worked per week & & & & & & $<0.001$ \\
\hline$<48 \mathrm{~h}$ & $21 / 213$ & $9.9(6.2-14.7)$ & $6 / 127$ & $4.7(2.2-11.0)$ & 1 & \\
\hline $48-60 \mathrm{~h}$ & $148 / 213$ & $69.5(62.8-75.6)$ & $71 / 127$ & $55.9(46.8-64.7)$ & $1.68(0.69-4.74)$ & \\
\hline$>60 \mathrm{~h}$ & $44 / 213$ & $20.6(15.4-26.7)$ & $50 / 127$ & $39.4(30.8-48.4)$ & $3.98(1.55-11.65)$ & \\
\hline Change in professional activity due to the COVID-19 outbreak & & & & & & 0.24 \\
\hline No & $39 / 213$ & $18.3(13.4-24.1)$ & $30 / 127$ & $23.6(16.5-32.0)$ & 1 & \\
\hline Yes & $174 / 213$ & $81.7(75.8-86.6)$ & $97 / 127$ & $76.4(68.0-83.5)$ & $0.72(0.42-1.25)$ & \\
\hline Direct involvement in the care of patients with COVID-19 & & & & & & 0.52 \\
\hline No & $125 / 213$ & $58.7(51.8-65.3)$ & $79 / 127$ & $62.2(53.1-70.7)$ & 1 & \\
\hline Yes & $88 / 213$ & $41.3(34.6-48.2)$ & $48 / 127$ & $37.8(29.3-46.8)$ & $0.86(0.55-1.35)$ & \\
\hline ICU admissions for COVID-19 per 100,000 inhabitants & & & & & & 0.46 \\
\hline$[0-0.5]$ & $45 / 213$ & $21.2(15.8-27.2)$ & $10 / 127$ & $15.0(3.8-14.0)$ & 1 & \\
\hline$[0.5-1]$ & $64 / 213$ & $30.0(24.0-36.7)$ & $44 / 127$ & $34.6(26.4-43.6)$ & $1.63(0.85-3.19)$ & \\
\hline$[1-2]$ & $49 / 213$ & $23.0(17.5-29.2)$ & $27 / 127$ & $21.3(14.5-29.4)$ & $1.31(0.64-2.69)$ & \\
\hline$>2$ & $55 / 213$ & $25.8(20.0-32.2)$ & $37 / 127$ & $29.1(21.4-37.9)$ & $1.59(0.81-3.18)$ & \\
\hline Anxiety score before the COVID-19 outbreak, median (IQR) & 35 & $(20-60)$ & 50 & $(40-70)$ & $1.15(1.09-1.21)$ & $<0.001$ \\
\hline Anxiety score during the COVID-19 outbreak, median (IQR) & 40 & $(25-60)$ & 50 & $(30-70)$ & $1.02(1.01-1.03)$ & $<0.001$ \\
\hline
\end{tabular}

$I Q R$, interquartile range; $C I$, confidence interval

* Intensive care unit admission rate for COVID-19 per 100,000 inhabitants in the resident's geographic area of practice (April 22 to 28, 2020)

generally accepted psychopathological subdimensions $[9,14$, $15,18,19]$. However, we used the MBI-HSS (a validated scale for assessing all three domains of burnout) and applied a common definition of burnout (a level of emotional exhaustion or depersonalization exceeding the usual cut-off) $[14,15]$. Secondly, our dataset came from a sample of 340 paediatric residents in France. In 2019, 325 new positions for paediatric residents became available, and the paediatric residency program lasts for 4 years. Although not all the available positions are filled and the deadline for replies was quite short, the response rate of $26.1 \%$ ( 340 out of $\sim 1300$ residents) was relatively high when compared with previous studies [19], and all the French university hospitals were represented. To the best of our knowledge, the present study was the first to have assessed burnout among paediatric residents in France (or in any European country, for that matter). Thirdly, our evaluation of the workload related to the COVID-19 outbreak was not very detailed. Nevertheless, we used three different items to try to increase the assessment's sensitivity. The first two items were two "yes"/"no" questions about the resident's professional activity. The third item was a quantitative question about the incidence of intensive care unit hospitalizations for COVID-19 in the week prior to the questionnaire being sent out. Fourthly, the study's cross-sectional design prevented us from defining causal relationships between the sociodemographic data, the prevalence of burnout, and 
COVID-19. The findings must be confirmed in a longitudinal study with measurements before and after a natural disaster. Further longitudinal study of risk factors associated with burnout (such as chronic exposure to stress) is also required [19].

Investigating the prevalence of burnout among the health care staff repeatedly (and not only after natural disasters) would help to focus attention on the health authorities' decisions with regard to work organization, supervision, time management, and stress [22]. Furthermore, support programs could usefully reduce work-related anxiety and could be monitored by regular assessing the prevalence of burnout - a major issue for both residents and the patients they care for [8]. Similar prospective studies in other European countries would enable comparisons of various health systems.

\section{Conclusion}

The prevalence $[95 \% \mathrm{CI}]$ of burnout among paediatric residents in France was 37.4\% [32.2-42.7]; 23.5\% [19.1-28.4] of the respondents reported a high level of emotional exhaustion, and 28.2\% [23.5-33.3] reported a high degree of depersonalization. Burnout was significantly associated with working hours per week and anxiety scores but not with exposure to the consequences of COVID-19; the latter observation might reflect the low incidence of symptomatic COVID-19 in children. Regular assessment of the prevalence of burnout within hospitals in France and in other countries might help to identify risk factors or protective factors for burnout; this would benefit both individuals and health systems/ organizations.

Supplementary Information The online version contains supplementary material available at https://doi.org/10.1007/s00431-020-03907-x.

Acknowledgements We thank the study participants for completing the questionnaire.

Authors' Contributions LT and PT contributed to the study conception and design. Material preparation, data collection, and analysis were performed by LT and PT. The first draft of the manuscript was written by LT and LT and PT commented on previous versions of the manuscript. LT and PT read and approved the final manuscript. PT supervised the study.

Data availability Data are not available for publication.

\section{Compliance with ethical standards}

Conflict of interest The authors declare that they have no conflict of interest.

Ethics approval The study protocol was approved by an independent ethics committee (Amiens University Hospital, Amiens, France; reference: PI2020 8430037 (April 10th, 2020).

Consent to participate N/A
Consent for publication N/A

Code availability All analysis was performed using R software (version 3.5.0), code is available on request.

\section{References}

1. Rotenstein LS, Ramos MA, Torre M, Segal JB, Peluso MJ, Guille C, Sen S, Mata DA (2016) Prevalence of depression, depressive symptoms, and suicidal ideation among medical students. JAMA. 316(21):2214-2236

2. Kansoun Z, Boyer L, Hodgkinson M, Villes V, Lançon C, Fond G (2019) Burnout in French physicians: a systematic review and meta-analysis. J Affect Disord 246:132-147

3. ICD-11 - Mortality and Morbidity Statistics [Internet]. [cited 2020 Jul 7]. Available from: https://icd.who.int/browse11/1-m/en\#/http:// id.who.int/icd/entity/129180281

4. Maslach C, Jackson SE, Leiter MP (2016) Maslach burnout inventory manual, 4th edn. Mind Garden Inc, Menlo Park

5. West CP, Dyrbye LN, Shanafelt TD (2018) Physician burnout: contributors, consequences and solutions. J Intern Med 283(6): 516-529

6. Whittaker E, Sinha R (2020) COVID-19: lessons learned from a paediatric high consequence infectious diseases unit. Arch Dis Child:archdischild-2020-319114. https://doi.org/10.1136/ archdischild-2020-319114

7. Fahrenkopf AM, Sectish TC, Barger LK, Sharek PJ, Lewin D, Chiang VW, Edwards S, Wiedermann BL, Landrigan CP (2008) Rates of medication errors among depressed and burnt out residents: prospective cohort study. BMJ. 336(7642):488-491

8. Halbesleben JRB, Rathert C (2008) Linking physician burnout and patient outcomes: exploring the dyadic relationship between physicians and patients. Health Care Manag Rev 33(1):29-39

9. Pantaleoni JL, Augustine EM, Sourkes BM, Bachrach LK (2014) Burnout in pediatric residents over a 2-year period: a longitudinal study. Acad Pediatr 14(2):167-172

10. Shah K, Chaudhari G, Kamrai D, Lail A, Patel RS (2020) How essential is to focus on physician's health and burnout in coronavirus (COVID-19) pandemic? Cureus 12(4):e7538

11. Wolpe J (1969) The practice of behavior therapy. Pergamon Press, London

12. Maslach C, Jackson SE (1981) The measurement of experienced burnout. J Organ Behav 2(2):99-113

13. Dion G, Tessier R (1994) Validation de la traduction de l'inventaire d'épuisement professionnel de Maslach et Jackson. Rev Can Sci Comport 26:210-227

14. Schaufeli WB, Bakker AB, Hoogduin K, Schaap C, Kladler A (2001) On the clinical validity of the maslach burnout inventory and the burnout measure. Psychol Health 16(5):565-582

15. Dyrbye LN, West CP, Shanafelt TD (2009) Defining burnout as a dichotomous variable. J Gen Intern Med 24(3):440

16. R Core Team (2013). R: a language and environment for statistical computing. R Foundation for statistical computing, Vienna, Austria http://www.R-project.org/

17. Kayloni O, Kathi JK, John DM (2015) What factors promote resilience and protect against burnout in first-year pediatric and medicine-pediatric residents? Journal of Evidence-Based Complementary \& Alternative Medicine 20(3):192-198

18. Baer TE, Feraco AM, Tuysuzoglu Sagalowsky S, Williams D, Litman HJ, Vinci RJ (2017) Pediatric resident burnout and attitudes toward patients. Pediatrics. 139(3):e20162163

19. Rodrigues H, Cobucci R, Oliveira A, Cabral JV, Medeiros L, Gurgel K, Souza T, Gonçalves AK (2018) Burnout syndrome 
among medical residents: a systematic review and meta-analysis. PLoS One 13(11):e0206840

20. Rushing CJ, Casciato DJ, Ead JK et al (2020) Perceptions of burnout, personal achievement, and anxiety among US podiatric medicine and surgery residents: a cross-sectional pilot study. J Foot Ankle Surg 000(2020):1-4

21. Paiva LC, Canario AC, China EL, Goncalves AK (2017) Burnout syndrome in health-care professionals in a university hospital. Clinics. 72(5):305-309

22. Mattei A, Fiasca F, Mazzei M, Necozione S, Bianchini V (2017) Stress and burnout in health-care workers after the 2009 L'Aquila earthquake: a cross-sectional observational study. Front Psychiatry 8:98

23. Yeo CJJ, Román GC, Kusnerik D, Burt T, Mersinger D, Thomas S, Boone T, Powell SZ (2018) Trainee responses to Hurricane Harvey: correlating volunteerism with burnout. Front Public Health 6:224
24. Metregiste D, Boucaud-Maitre D, Aubert L, Noubou L, Jehel L (2020) Explanatory factors of post-traumatic distress and burnout among hospital staff 6 months after Hurricane Irma in Saint-Martin and Saint-Barthelemy. PLoS One 15(3):e0229246

25. Wang Z, Zhou Q, Wang C, Shi Q et al (2020) Clinical characteristics of children with COVID-19: a rapid review and meta-analysis. Ann Transl Med 8(10):620

26. Dimitriu MCT, Pantea-Stoian A, Smaranda AC, Nica AA, Carap AC, Constantin VD, Davitoiu AM, Cirstoveanu C, Bacalbasa N, Bratu OG, Jacota-Alexe F, Badiu CD, Smarandache CG, Socea B (2020) Burnout syndrome in Romanian medical residents in time of the COVID-19 pandemic. Med Hypotheses 144:109972

Publisher's note Springer Nature remains neutral with regard to jurisdictional claims in published maps and institutional affiliations. 